\title{
CARGA ORGANICA DE LAS AGUAS NEGRAS MUNICIPALES DE LA CIUDAD DE TIJUANA, BAJA CALIFORNIA
}

\author{
Por \\ Sergio Adolfo Sañudo Wilhelmy \\ Instituto de Investigaciones Oceanológicas \\ Universidad Autónoma de Baja California \\ Apartado Postal No. 453 \\ Ensenada, Baja California \\ $\mathbf{y}$ \\ Carlos Eugenio Suárez Vidal \\ Dirección de Pesca del Estado \\ Ave. Ruiz No. 4-3 Altos \\ Ensenada, Baja California
}

\section{RESUMEN}

Con el propósito de evaluar la carga orgánica de la Ciudad se realizaron 88 muestreos en el colector principal de las aguas negras de la Comisión Estatal de Servicios Públicos de Tijuana (C.E.S.P.T.) en el período comprendido en tre febrero de 1979 a enero de 1980 . Se realizaron análisis para la demanda bioquímica de oxígeno (DBO) grasa y aceites (G y A) sólidos totales (ST), sólidos suspendidos (SST), sólidos sedimentales (SS), potencial hidrógeno $(\mathrm{pH})$ y temperatura $(\mathrm{T})$.

La carga orgánica promedio diario encontrada fue de 7 toneladas para la $\mathrm{DBO}, 15$ toneladas para la DQO, y 42 toneladas para los sólidos totales. Se realizó la predicción de la carga orgánica promedio diaria esperada para el año 2000. Esta fue de 34 toneladas para la DBO, 71 para la DQO y de 199 toneladas para los sólidos totales. Los valores encontrados exceden a los límites permitidos por la Legislación Mexicana, desde 161 o/o en G y A hasta 1045 o/o en SS. Los valores de temperatura y $\mathrm{pH}$ estuvieron dentro del límite permitido.

\section{ABSTRACT}

From February 1979 to January 1980 we sampled the wastewater from the principal sewage collector of urban waters of the city of Tijuana, B.C., operated by the Comision Estatal de Servicios Publicos (CESP). The main objective was to evaluate the organic load of the city as to its biochemical oxygen demand (BOD), chemical oxygen demand (COD), oil and grease, total solids, suspended solids, settleable solids, $\mathrm{pH}$ and temperature.

It was found that the mean average daily load for the BOD was 7 tons, 15 tons for the COD, and 42 tons for total solids. Based on extrapolation of sewage flow, we estimated that by the year 2000 urban wastes would reach 34 tons for BOD, 71 tons for COD, and 199 tons for total solids. 
The values found this work for oil and grease and settleable solids exceed by $161 \%$ o to $1045 \%$ o respectively the permitted levels stated in the Mexican Legislation.

\section{INTRODUCCION}

De acuerdo a la organización Panamericana de la Salud (1976), las descargas Municipales de aguas negras son consideradas como una fuente importante de contaminación en las zonas costeras. Dichas aguas se caracterizan por contener altas concentraciones de materia orgánica que provee un recurso de material y energía para los organismos heterotróficos principalmente bacterias, las cuales son responsables en parte de la degradación oxidativa del material orgánico (Cronin y Flemer, 1967; Warren, 1971).

Golubic (1970); Dugan (1972); McIntyre y Murison (1972); Christie y Moldan (1977), establecen que la introducción de la materia orgánica al medio marino puede ocasionar un decremento en la penetración de la luz, afectando la razón entre fotosíntesis y respiración, así como cambios en la abundancia y diversidad de las poblaciones, principalmente bentónicas, pudiendo altẹrar los flujos de energía entre especies.

De acuerdo a Suárez (1974), la calidad y cantidad de estas aguas de desecho están en función del desarrollo económico y social de la población que la genera. Lo anterior se demuestra a través del trabajo de Wolman (1969), el cuál calculó en los Estados Unidos que por cada millón de habitantes se generaba un agua residual que contenía alrededor de 110 toneladas de sólidos suspendidos diarios. En el sur del Estado de California, E.U.A. en 1977 el flujo de las 5 principales descargas, promedió 3600 millones de litros por día conteniendo un promedio diario de 650 toneladas de sólidos (Schafer, 1978).

En México, las industrias tanto como los conglomerados humanos, se localizan en su mayor parte en la región central del país, lo que favorece la ausencia de fuertes contaminaciones de las aguas marinas. En años recientes, este panorama ha cambiado, debido al impulso económico que le ha dado a los Estados localizados en las zonas costeras, entre ellos al de Baja Califormia, y particularmente a la Ciudad de Tijuana.

Tomando en cuenta lo anterior y a que no se conoce de un trabajo similar en este lugar, el presente estudio tiene como objetivos el de estimar la carga orgánica generada por el 43 o/o de la población de Tijuana, B.C. (269,585 habitantes) que en 1979 contaba con servicio de alcantarillado (Negrete et al., 1980). Comparar los valores encontrados con los valores máximos permitidos para la calidad del efluente establecidos por la Legislación de México y de otros países. Realizar una predicción de la calidad de los desechos esperados a futuro. 


\section{MATERLALES Y METODOS}

Los muestreos fueron realizados en el colector principal de las aguas residuales de la Comisión Estatal de Servicios Públicos de Tijuana (C.E.S.P.T.), del mes de febrero de 1979 a enero de 1980 . Efectuándose un total de 11 variaciones semidiurnas (6:00 - 18:00 horas) durante este período de tiempo. Las muestras obtenidas para el análisis de la demanda bioquímica de oxígeno (DBO), demanda química de oxígeno (DQO), grasas y aceites (G y A), sólidos totales (ST), sólidos totales volátiles (STV), sólidos totales fijos (STF), sólidos suspendidos totales (SST), sólidos suspendidos volátiles (SSV), y sólidos suspendidos fijos (SSF), fueron almacenadas a bajo temperatura $\left(0^{\circ} \mathrm{C}\right)$, para su posterior análisis en laboratorio. La temperatura $\left(\mathrm{T}^{\circ} \mathrm{C}\right)$, el potencial hidrógeno $(\mathrm{pH})$ y los sólidos sedimentales (SS), se determinó "in situ ". Las técnicas de muestreo y de análisis fueron de acuerdo al manual de la American Public Health Association (1975).

La localización del área de estudio para la estimación de la carga orgánica de la Ciudad de Tijuana, B.C., se representa en la Figura 1.

\section{RESULTADOS Y DISCUSION}

La media anual para cada parámetro se muestra en la Tabla 1, como la característica promedio anual de las aguas residuales de la Ciudad de Tijuana, B.C. Estos valores, se compararon con las características promedio de las aguas residuales municipales para la zona norte y centro del país de acuerdo al Plan Nacional Hidráulico de 1976. (Secretaría de Recursos Hidráulicos 1976) (Tabla 2), encontrándose, que la Ciudad de Tijuana presenta valores promedio similares a la zona centro que se considera de mayor contaminación.

Los valores de la media anual al multiplicarse por el gasto promedio (500 litros por segundo en 1979, comunicación personal con el Ing. Manuel Becerra, Superintendente de Operación CESPT y por el tiempo hora, dias, mes) se obtuvo una carga orgánica promedio en toneladas por día , toneladas por mes y toneladas por año (de febrero de 1979 a enero de 1980) (Tablas 3, 4 y 5). El coeficiente de variación estimado para la DBO (Tablas 1,3 y 4) fue entre el 15 y 17 o/o que de acuerdo a Velz (1976), se presenta en ciudades grandes.

Los valores de la DQO fueron mayores que los de la $\mathrm{DBO}$, siendo la relación DBO:DQO de 1:2.11 respectivamente. De acuerdo a Ford (1976), el consumo de oxígeno por la DQO en este tipo de agua es mayor que el de la DBO, esto se debe a que las aguas negras son una mezcla de sustancias orgánicas e inorgánicas donde la DQO medirá el consumo de oxígeno de una parte de la porción orgánica más el consumo de la inorgánica, mientras que la DBO únicamente mide el consumo de la parte orgánica (Mancy, 1972).

En el análisis de las descargas deben de considerarse las condiciones a futuro 
CARGA ORGANICA DE LAS AGUAS NEGRAS

Tabla 2.- Características promedio de las aguas residuales municipdes para la zona Norte y Centro del País así como de la Ciudad de Tijuana, B. C.

\begin{tabular}{|l|r|r|c|}
\hline \multicolumn{1}{|c|}{ PARAMETROS } & NORTE* & $\begin{array}{c}\text { Z O N A S } \\
\text { CENTRO* }\end{array}$ & TIJUANA, B.C. \\
\hline pH (unidades) & 7.1 & 7.2 & 7.3 \\
ToC & 15.4 & 25.8 & 21.5 \\
DBD mg/1 & 229.2 & 325.7 & 325.78 \\
DOO Mg/1 & 461.9 & 648.1 & 688.34 \\
Sólidos Sed. ml/1 & 1.6 & 8.7 & 10.45 \\
G y A mg/1 & 45.7 & 60.3 & 115.66 \\
Sólidos Tot. mg/1 & 1335.6 & 1115.2 & 1919.57 \\
Sólidos Tot. Vol. mg/1 & 443.1 & 524.2 & 689.09 \\
Sólidos Tot. F. mg/1 & 919.7 & 617.4 & 1060.62 \\
Sólidos Susp. Tot. $\mathrm{mg} / 1$ & 218.9 & 316.4 & 384.19 \\
Sólidos Susp. Vol. $\mathrm{mg} / 1$ & 114.0 & 228.2 & 256.60 \\
Sólidos Susp. Fij. $\mathrm{mg} / 1$ & 105.0 & 106.0 & 127.59 \\
\hline
\end{tabular}

* Valores promedio de la calidad de las aguas negras de acuerdo al Plan Nacional Hidráulico (S.R.H. 1976). 
Tabla 1.- Característica promedio anual de la calidad de las aguas residuales de la Ciudad de Tijuana, B. C. (Todos en miligramos por litro con excepción de los sólidos sedimentables en mililitros por litro).

\begin{tabular}{|c|c|c|c|c|c|c|c|c|}
\hline \multirow{3}{*}{\begin{tabular}{|l} 
PARAMETROS \\
Temperatura \\
pH
\end{tabular}} & \multirow{3}{*}{$\begin{array}{l}n \\
11 \\
11\end{array}$} & \multirow{2}{*}{$\begin{array}{r}\text { MEDIA } \\
21.5739\end{array}$} & \multirow{2}{*}{$\begin{array}{l}\text { DESVIACION } \\
\text { ESTANDAR } \\
3.2400\end{array}$} & \multicolumn{2}{|c|}{$\begin{array}{c}\text { R A NGO } \\
\text { MINIMO MAXIMO }\end{array}$} & \multicolumn{2}{|c|}{$\begin{array}{l}\text { LIMITE DE CONFIANZA } \\
\text { DE LA MEDIA A } 950 / 0\end{array}$} & \multirow{2}{*}{$\begin{array}{c}\begin{array}{c}\text { COEFICIENTE } \\
\text { de VARIACION } \\
\text { (o/o) }\end{array} \\
15.02\end{array}$} \\
\hline & & & & 15.94 & 25.80 & 19.3972 & 23.7483 & \\
\hline & & 7.3318 & 0.2660 & 7.00 & 7.93 & 7.1530 & 7.5106 & 3.63 \\
\hline Oxígeno Disuelto & 11 & 0.4845 & 0.9890 & 0.00 & 3.37 & 00.0000 & 1.1494 & 204.13 \\
\hline DBO & 11 & 324.8700 & 54.3000 & 284.39 & 462.33 & 288.3519 & 361.3806 & 16.71 \\
\hline BOO & 11 & 688.3400 & 125.0000 & 524.03 & 918.74 & 604.1702 & 772.5004 & 18.16 \\
\hline Grasas y Aceites & 10 & 115.6640 & 12.7139 & 92.02 & 130.80 & 106.1702 & 124.5004 & 10.99 \\
\hline Sólidos Sediment. & 11 & 10.5140 & 2.9400 & 6.60 & 17.50 & 8.5400 & 12.4873 & 17.96 \\
\hline Sólidos totales & 11 & 1919.6000 & 127.0000 & 1810.52 & 2276.14 & 1833.9929 & 2005.1392 & 6.62 \\
\hline Sólidos Tot. Vol. & 10 & 758.0070 & 81.7822 & 687.58 & 959.71 & 699.5580 & 816.4560 & 10.79 \\
\hline Sólidos Tot. Fijos & 10 & 1171.0790 & 72.8741 & 1065.13 & 1316.43 & 1118.9964 & 1223.1616 & 6.22 \\
\hline Sólidos Susp. Tot. & 8 & 384.1875 & 67.1001 & 277.21 & 460.92 & 328.1996 & 440.1754 & 17.47 \\
\hline Sólidos Susp. Vol. & 8 & 256.6013 & 69.3865 & 195.50 & 362.30 & 198.7056 & 314.4970 & 27.04 \\
\hline Sólidos Susp. Fijos & 8 & 127.5988 & 63.7786 & 60.97 & 260.59 & 74.3822 & 180.8154 & 49.98 \\
\hline
\end{tabular}


de los desechos, estas condiciones están en función de dos factores como son el incremento de la población y el desarrollo industrial (Velz, 1976). El primero ocasionando cambios en el gasto de la descarga y el segundo alterando las características primarias del desecho (Auld, 1976).

La Tabla No. 6 muestra una estimación de los valores esperados de la carga orgánica promedio en toneladas por día para los años de 1982 y 2000 . Esto se realizó suponiendo que las características primarias de los desechos no varien y lo único que cambie sea el gasto.

Aunque la descarga al mar no se realiza en el lugar de muestreo, los valores de la característica promedio anual de las aguas residuales de la ciudad de Tijuana, B.C., se compararon con los valores máximos permitidos para la calidad de efluente establecidos por las Legislaciones de México (S.R.H., 1973) California, U.S.A. (Pearson, 1973) y Singapur (Ludwig, 1973), notándose que la Legislación Mexicana no presenta valores para la DBO y DQO. La legislación con valores más altos es también la de México (Tabla 7).

Los porcentajes sobre el límite permitidoen que se encuentran las aguas residuales de Tijuana, B.C., con respecto a las legislaciones citadas se presentan en la Tabla 8, observándose que los sólidos sedimentales (SS) fue el parámetro que más se excedió, en México 1045 o/o; en California,U.S.A. 5225 o/o y en Singapur 2090 o/o. La DBO estuvo 724 o/o con respecto a la de California y 652 o/o con la de Singapur.

Con la información obtenida se calculó un factor de dilución para las aguas negras de la Ciudad, siendo 80 veces $(1: 80)$ (Tabla 9), en un cuerpo de agua cerrado al cual permitirá conservar únicamente un valor de 5 miligramos por litro de oxígeno disuelto, que es el límite permitido por la Legislación Mexicana para un cuerpo de agua receptor para uso recreativo (S.R.H., 1973).

\section{CONCLUSIONES}

De los valores obtenidos en la Ciudad de Tijuana, B.C, se concluye que el agua residual estudiada se caracteriza por tener un alto contenido de material orgánico que requiere de un gran consumo de oxígeno. Estos valores se encuentran muy por encima de los establecidos por la Legislación Mexicana y por la de otros países. La Legislación de nuestro país tiene un rango muy amplio y no toma en cuenta parámetros como DBO y DQO, lo cual permite que las concentraciones de material orgánico que se pudiera vertir en los cuerpos receptores sean mayores. Los volúmenes de los desechos esperados para el año 2000 son muy altos, lo que ocasionará serios problemas en la disposición de los desechos. 
Tabla 3.- Valores promedio diario de la carga orgánica de la Ciudad de Tijuana, B. C., en toneladas por día

\begin{tabular}{|c|c|c|c|c|c|c|c|c|}
\hline PARAMETROS & $n$ & MEDIA & $\begin{array}{l}\text { DESVIACION } \\
\text { ESTANDAR }\end{array}$ & $\begin{array}{r}\text { RA I } \\
\text { MINIMO }\end{array}$ & $\begin{array}{l}0 \\
\text { AXIMO }\end{array}$ & $\begin{array}{l}\text { LIMITE DE } \\
\text { DE LA MEI }\end{array}$ & $\begin{array}{l}\text { FIANZA } \\
95 \%\end{array}$ & $\begin{array}{l}\text { COEFICIENTE } \\
\text { de VARIACION }\end{array}$ \\
\hline DBO & 11 & 7.0354 & 1.1764 & 6.14 & 9.99 & 6.2444 & 7.8264 & 16.72 \\
\hline DQO & 11 & 14.8673 & 2.7043 & 11.32 & 19.84 & 13.0490 & 16.6856 & 18.19 \\
\hline Grasas y Aceites & 10 & 2.4970 & 0.2726 & 1.99 & 2.83 & 2.3022 & 2.6918 & 10.91 \\
\hline $\begin{array}{l}\text { Sólidos totales } \\
\text { Volátiles }\end{array}$ & 10 & 16.3730 & 1.7673 & 14.85 & 20.73 & 15.1099 & 17.6361 & 10.80 \\
\hline $\begin{array}{l}\text { Sólidos totales } \\
\text { Fijos }\end{array}$ & 10 & 25.2970 & 1.5716 & 23.00 & 28.43 & 24.1738 & 26.4200 & 6.21 \\
\hline Sólidos Susp. Tot. & 8 & 8.2963 & 1.4571 & 5.98 & 9.97 & 7.0804 & 9.5122 & 17.56 \\
\hline Sólidos Susp. Vol. & 8 & 5.5413 & 1.5013 & 3.20 & 7.83 & 4.2886 & 6.7940 & 27.09 \\
\hline Sólidos Susp. Fijos & 8 & 2.7550 & 1.2782 & 1.21 & 6.63 & 1.6050 & 3.9050 & 50.02 \\
\hline
\end{tabular}


Tabla 4.- Valores promedio mensual de la carga orgánica de la Ciudad de Tijuana, B. C., en toneladas por mes.

\begin{tabular}{|c|c|c|c|c|c|c|c|c|}
\hline \multirow{2}{*}{$\begin{array}{l}\text { PARAMETROS } \\
\text { DBO }\end{array}$} & \multirow{2}{*}{$\begin{array}{l}n \\
\\
11\end{array}$} & \multirow{2}{*}{$\begin{array}{r}\text { MEDIA } \\
182.3100\end{array}$} & \multirow{2}{*}{$\begin{array}{l}\text { DESVIACION } \\
\text { ESTANDAR } \\
28.7200\end{array}$} & \multicolumn{2}{|c|}{$\begin{array}{c}\text { R A NGO } \\
\text { MINIMO MAXIMO }\end{array}$} & \multicolumn{2}{|c|}{$\begin{array}{l}\text { LIMITE DE CONFIANZA } \\
\text { DE LA MEDIA A } 95 \text { \%/0 }\end{array}$} & \multirow{2}{*}{\begin{tabular}{|c}
$\begin{array}{c}\text { COEFICIENTE } \\
\text { de VARIACION } \\
(0 / 0)\end{array}$ \\
15.75
\end{tabular}} \\
\hline & & & & 156.57 & 249.75 & 162.9994 & 201.6207 & \\
\hline DQO & 11 & 385.8200 & 70.3000 & 271.68 & 505.92 & 338.5521 & 433.0877 & 18.22 \\
\hline Grasas y Aceites & 10 & 64.8240 & 6.5923 & 53.73 & 72.90 & 60.1126 & 69.5354 & 10.17 \\
\hline Sólidos Totales & 11 & 1075.2691. & 66.6811 & 1004.19 & 1253.58 & 1030.4343 & 1120.1039 & 6.20 \\
\hline Sólidos Tot. Vol. & 10 & 425.5040 & 45.8831 & 365.76 & 528.61 & 392.7128 & 458.2952 & 10.78 \\
\hline Sólidos Tot. Fijos & 10 & 656.8730 & 32.2861 & 609.60 & 724.97 & 633.7991 & 679.9469 & 4.92 \\
\hline Sólidos Susp. Tot. & 8 & 217.1000 & 37.5508 & 152.49 & 264.34 & 185.7679 & 248.4321 & 17.30 \\
\hline Sólidos Susp. Vol. & 8 & 145.9900 & 42.9340 & 81.60 & 211.41 & 110.1661 & 181.8128 & 29.41 \\
\hline Sólidos Susp. Fijos & 8 & 71.1350 & 32.2492 & 35.64 & 135.12 & 44.2265 & 98.0435 & 45.34 \\
\hline
\end{tabular}


Tabla No. 5 Carga orgánica de las aguas residuales de la ciudad de Tijuana, B.C., durante febrero de 1979 a enero de 1980.

\begin{tabular}{|l|r|}
\hline PARAMETROS & TONELADAS \\
\hline & \\
Demanda Bioquimica de Oxígeno & \\
Demanda Ouímica de Oxigeno & $2,005.37$ \\
Grasas y Aceites & $4,244.01$ \\
Sólidos Totales & 684.24 \\
Sólidos Totales Volátiles & $1,827.96$ \\
Sólidos Totales Fijos & $4,255.04$ \\
Sólidos Suspendidos Totales & $6,568.73$ \\
Sólidos Suspendidos Volatiles & $1,736.80$ \\
Sólidos Suspendidos Fijos & $1,167.92$ \\
\hline
\end{tabular}

Tabla 6. Carga orgánica promedio esperada para el año de 1982 y 2000 y 2000 de las aguas residuales en la ciudad de Tijuana, B.C. (gastos esperados en litros por segundo (1/seg.) proporcionados.

\begin{tabular}{|l|c|c|}
\hline \multicolumn{3}{|c|}{ CARGA PROMEDIO EN TONELADAS / DIA } \\
\hline & 1982 (800 1/Seg.) & $2000(24001 / \mathrm{Seg})$. \\
\cline { 2 - 3 } Demanda Bioquímica de Oxígeno & 11.26 & 33.78 \\
Demanda Química de Oxígeno & 23.79 & 71.37 \\
Grasas y Aceites & 3.91 & 11.73 \\
Sólidos Totales & 66.34 & 199.02 \\
Sólidos Totales Volạ́tiles & 23.81 & 71.43 \\
Sólidos Totales Fijos & 36.66 & 109.98 \\
Sólidos Suspendidos Totales & 13.28 & 39.84 \\
Sólidos Suspendidos Volátiles & 8.87 & 26.61 \\
Sólidos Suspendidos Fijos & 4.41 & 13.23 \\
\hline
\end{tabular}


Tabla 7.- Valores máximos permitidos de la calidad deñ efluente en Mézico, California, E.U.A. y Singapur.

\begin{tabular}{|l|c|c|c|}
\hline \multicolumn{1}{|c|}{ PARAMETROS } & MEXICO* & $\begin{array}{c}\text { CALIFORNIA } \\
\text { E.U.A.** }\end{array}$ & SINGAPUR *** $^{* *}$ \\
\hline $\mathrm{T}^{\circ} \mathrm{C}$ & 35 & - & - \\
$\mathrm{pH}$ & $4.5-10$ & $6-9$ & $6-9$ \\
Grasas y Aceites $\mathrm{mg} / 1$ & 70.0 & 5.0 & 5.0 \\
Sólidos Sedimentables ml/1 & 1.0 & 0.2 & 0.5 \\
DBO mg/1 & - & $30-45$ & 50.0 \\
DOO Mg/1 & - & - & 100.0 \\
\hline
\end{tabular}

- Secretaria de Recursos Hidráulicos, 1973.

* Pearson, 1973

"** Ludwig, 1973

Tabla 8.- Porcentajes sobre el límite permitido en que se encuentran las aguas residuales de la Ciudad de Tijuana, B. C., con respecto a las legislaciones de México, California, U.S.A. y Singapur.

\begin{tabular}{|l|c|c|c|}
\hline \multicolumn{1}{|c|}{ PARAMETRO } & MEXICO & $\begin{array}{c}\text { CALIFORNIA } \\
\text { E.U.A. }\end{array}$ & SINGAPUR \\
\hline $\mathrm{T}^{\mathrm{o}} \mathrm{C}$ & $/$ & - & - \\
$\mathrm{pH}$ & $/$ & $/$ & $/$ \\
Grasas y Aceites & $161 \mathrm{o} / \mathrm{o}$ & $2260 \mathrm{o} / \mathrm{o}$ & $2260 \mathrm{o} / \mathrm{o}$ \\
Sólidos Sediment. & $1045 \mathrm{o} / \mathrm{o}$ & $5225 \mathrm{o} / \mathrm{o}$ & $2290 \mathrm{o} / \mathrm{o}$ \\
DBO & - & $724 \mathrm{o} / \mathrm{o}$ & $652 \mathrm{o} / \mathrm{o}$ \\
DOO & - & - & $688 \mathrm{o} / \mathrm{o}$ \\
\hline
\end{tabular}


Tabla 9.- Factor de dilución para las aguas residuales de la Ciudad de Tijuana, B. C.

/ Parámetro dentro del límite permitido.

\section{FACTOR DE DILUCION - 80 VECES}

PARA:

- Un gasto de descarga de $5001 /$ Seg.

- Una concentración de oxígeno disuelto en el cuerpo receptor de $9 \mathrm{mg} / 1$.

- Un valor esperado de oxígenos disuelto en el cuerpo receptor de $5 \mathrm{mg} / 1$.

\section{BIBLIOGRAFIA}

American Public Health Association, American Water Works Association y Water Pollution Control Federation. 1975. Métodos estándar para el exámen de aguas y aguas de desecho. Edit. Interamericana. México, D.F. 11 ed. pp. 609.

Auld, D.V. 1976. Wastewater in the city. En: Handbook of water resources and pollution control. Harry W. Gehm y Jacob I. Bregman (eds.) Van Nostrand Reinhold, New York pp.,429-460.

Christie, N.D. y A. Moldan 1977. Effects of fish factory effluent on the benthic macrofauna of Saldanha Bay. Marine Pollution Bull. B(2):41-45.

Cronin, L.E. y D.A. Flemer. 1967. Energy transfer and pollution. En: T.A. Olson y F.J. Burgess (ed), Pollution and Marine Ecology. Interscience Publishers, New York, p.171-185.

Dugan, P.R. 1972. Biochemical ecology of water pollution. Plenum Press, New York, p. 7-8.

Ford, D.L. 1976. Water pollution control in the petroleum industry. En: Hardam Singh Azad (ed.), Industrial wastewater management handbook.

Mc Graw-Hill: New York, N.Y. pp.8.1-8.75.

Golubic, S. 1970. Effect of organic pollution on benthic communities. Marine Pollution Bull. INS (4): 56-57. 
Ludwig, H.F. 1973. Criteria for marine waste disposal in South-east Asia. En: E.A. Pearson y E. de Franja Franjipane. (eds.) Marine pollution and marine waste disposal. Pergamon Press, Oxford, pp. 99-108.

Mancy, H.K. 1972. Analytical problems in water pollution control. En: W.W. Meinke y J.K. Taylor (eds.) Analytical chemestry key to progress on national problems. Proccedings of the 24 th. Annual Summer Symposium on Anal. Chemistry, National Bureau of standards. Special Publ. 351. Washingnton, D.C. pp. 356-357.

McIntyre, A.D. and D.J. Murison. 1972. The benthos of a polluted beach. I.C.E.S.C.M. 1972/E:30, Fish Improvement Comm.

Negrete Hurtado, E., M. Pérez Delgado y E. Wilhelmy. 1980. Ordenamiento del territorio municipal de Tijuana. Tesis Profesional, Esc. de Arquitectura de la U.A.B.C.; Mexicali, B.C. 131 p.

Organización Panamericana de la Salud y Organización Mundial de la Salud.1976. Riesgos del Ambiente Humano para Ia Salud - Organización Mundial de la Salud y Organización Panamericana de la Salud. (Eds.).

Pearson, E.A. 1973. Criteria for marine waste disposal in California. En: Pearson, E.A. y E. de Fraja Franjipane. (eds), - Marine pollution and marine waste disposal.. Pergamon Press, Oxford. pp. 85-87.

Secretaría de Recursos Hidráulicos. 1973. Legislación relativa al agua y su contaminación. p. 143.

Secretaría de Recursos Hidráulicos. 1976. Protección y mejoramiento de la calidad del agua. Subsecretaría de Planeación. 1ra. Ed. 13:18-87.

Schafer, H.A. 1978. Characteristics of municipal wastewater discharges. En: Coastal Water Research Project,. Annual Rep. pp. 97-101.

Suárez, L. 1974. La Contaminación. Testimonios de Fondo, Ed. Fondo de Cultura Económica. México, 12, D.F. p. 10.

Velz, C.J. 1976. Stream analysis-forecasting waste assimilation capacity. En: Harry W. Gehm y Jacob I. Breagman (eds.) Handbook of water resources and pollution control. Van Nostrand Reinhold, New York, pp. 216-261.

Warren, E.C. 1971. Biology and water pollution control. Six kinds of water pollution. W.B. Saunders, Philadelphia, pp. 57-63.

Wolman, A. 1969. Water, Health and Society. Blomington, Ind., Indiana University Press. 